\title{
Simulations of 3D dynamics of microdroplets : a comparison of rectangular and cylindrical channels
}

\author{
Charles-Henri BRUNEAU, Thierry COLIN, Cédric GALUSINSKI, Sandra \\ TANCOGNE, and Paul VIGNEAUX*
}

\begin{abstract}
In this paper, several numerical simulations of diphasic flows in microchannels are presented. The flow in both cylindrical and rectangular channels is considered. The aim is to compute the shape of the droplets and the velocity fields inside and outside the droplets and to quantify the influence of the geometry. The Level Set method is used to follow the interface between the fluids.
\end{abstract}

\section{Introduction}

Diphasic flows in microchannels are governed by the pressure gradient and the surface tension at the interface between the fluids. In experimental configurations, jets of one fluid into another are usually not stable. This is due to Rayleigh instability. The jets can break off and therefore can lead to the creation of droplets. The study of the flow inside these droplets is a difficult task that can be achieved only with a numerical approach.

From the experimental point of view, once they are created, droplets propagate at their own speed while the flow between two droplets is essentially a Poiseuille flow,

Charles-Henri BRUNEAU

e-mail: bruneau@math.u-bordeaux1.fr

Thierry COLIN

e-mail: colin@math.u-bordeaux1.fr

Sandra TANCOGNE

e-mail: tancogne@math.u-bordeaux1.fr

* Corresponding author, Paul VIGNEAUX

Institut de Mathématiques de Bordeaux, Inria Bordeaux MC2 - 351 Cours de la Libération - F33405 Talence Cedex - France, e-mail: Paul.Vigneaux@math.cnrs.fr

Cédric GALUSINSKI

CPT-Imath, Université du Sud - Toulon Var - Avenue de l'Université - BP20132 83957 La Garde

Cedex - France, e-mail: galusins@univ-tln.fr 
as soon as the droplets are "sufficiently" separated. This implies that some recirculations occurs inside the droplets in order to ensure a non-slip boundary condition on the boundary of the channels. These movements are not only created by the flow itself but also by the surface tension forces that are responsible of the shape of the droplets. From the point of view of the applications these microdroplets can be used as micromixers and as microreactors in order to achieve reactions with very small volumes of products. One of the characteristics of microflows is that they are constrained by the confinement. Typical size of a microchannel is a section of $10^{-8} \mathrm{~m}^{2}$ with a length of a few centimeters. Typical velocity of the flows is $1 \mathrm{~cm} / \mathrm{s}$. The flow rates are around $3000 \mu \mathrm{l}$ per hour. This confinement determines strongly the stability of the jets or of the co-flows as well as the shape of the droplets in case of breakup. The geometry of the channels are also very important for the dynamics of the droplets. One can build and use cylindrical channels as well as rectangular ones. For very thin jets, localized in the middle of the channel, the shape of the section of the channel is not determinant and flows in cylindrical and rectangular channels have the same behavior. The explanation is that surface tension forces are important at these scales and the velocity field at the interface is closed to be invariant under rotations. This is observed on the numerical simulations of flows without confinement whatever the shape of the channel is. For larger structures, the flow undergoes the effects of confinement and circular and rectangular channels give rise to different kinds of behavior.

The aim of this note is to present a preliminary comparison of droplets and their associate flows in both cylindrical and rectangular channels. It is organized as follows. In the second part, we recall the model and the numerical method used. In the third part, we try to emphasize the main features of 3D flows in cylindrical and rectangular channels.

\section{Modelling and simulation of bifluid microflows}

\subsection{Governing equations}

We want to modelize incompressible, viscous and Newtonian bifluid flows with surface tension in microchannels. This requires to follow carefully the moving interfaces. We adopt a Level Set approach to capture the interface $\Gamma$ between fluid 1 and fluid 2 (see [7] and [6]). In this context, the interface is given by the zero level set of a function $\phi$ :

$$
\Gamma(t)=\{x \in \Omega / \phi(x, t)=0\}, \forall t \geq 0
$$

where $\Omega$ is the $3 \mathrm{D}$ bounded computational domain occupied by the fluids. Moreover $\phi$ satisfies 


$$
\forall t \geq 0,\left\{\begin{array}{l}
\phi(x, t)<0 \forall x \in \text { fluid } 1 \\
\phi(x, t)>0 \quad \forall x \in \text { fluid } 2 \\
\phi(x, t)=0 \quad \forall x \in \Gamma(t)
\end{array}\right.
$$

In the context of microfluidics, the Reynolds number is small and we can neglect the inertial effects. The velocity is then a solution of the Stokes equation

$$
\begin{gathered}
-\nabla \cdot(2 \eta D \mathbf{u})+\nabla p=\sigma \kappa \delta(\phi) \mathbf{n} \quad \forall(t, \mathbf{x}) \in \mathbb{R}^{+} \times \Omega, \\
\nabla \cdot \mathbf{u}=0 \quad \forall(t, \mathbf{x}) \in \mathbb{R}^{+} \times \Omega,
\end{gathered}
$$

where $\eta$ is the viscosity, $p$ is the pressure and $D \mathbf{u}=\left(\nabla \mathbf{u}+\nabla^{T} \mathbf{u}\right) / 2$ is the deformation rate tensor, $\sigma \in \mathbb{R}$ is the constant surface tension coefficient, $\mathbf{n}$ is the unit normal to the interface, $\kappa$ is the curvature of the interface and $\delta$ is the Dirac function. The normal and the curvature are computed thanks to the Level Set function :

$$
\begin{gathered}
\mathbf{n}=\left.\frac{\nabla \phi}{|\nabla \phi|}\right|_{\phi=0}, \\
\kappa=\left.\nabla \cdot\left(\frac{\nabla \phi}{|\nabla \phi|}\right)\right|_{\phi=0} .
\end{gathered}
$$

In addition, the viscosity is given by

$$
\eta=\eta_{1}+\left(\eta_{2}-\eta_{1}\right) H(\phi)
$$

where $\eta_{1}$ (resp. $\eta_{2}$ ) is the viscosity of fluid 1 (resp. 2) and $H$ is the Heaviside function:

$$
H(\phi)=\left\{\begin{array}{l}
0 \text { if } \phi \leq 0 \\
1 \text { if } \phi>0
\end{array}\right.
$$

The interface moves at the velocity of the fluid and the function $\phi$ is then defined [8] as the solution of the advection equation :

$$
\frac{\partial \phi}{\partial t}+\mathbf{u} . \nabla \phi=0 \quad \forall(t, \mathbf{x}) \in \mathbb{R}^{+} \times \Omega .
$$

Finally, two types of geometry are considered, namely rectangular channels and cylindrical channels, as shown on Figure 1.

\subsection{Numerical resolution procedure}

The numerical algorithm is the following one :

1. Compute an initial value for the Level Set function $\phi$ and related $\eta$.

2. Compute the unit normal $\mathbf{n}$ and the curvature $\kappa$. 

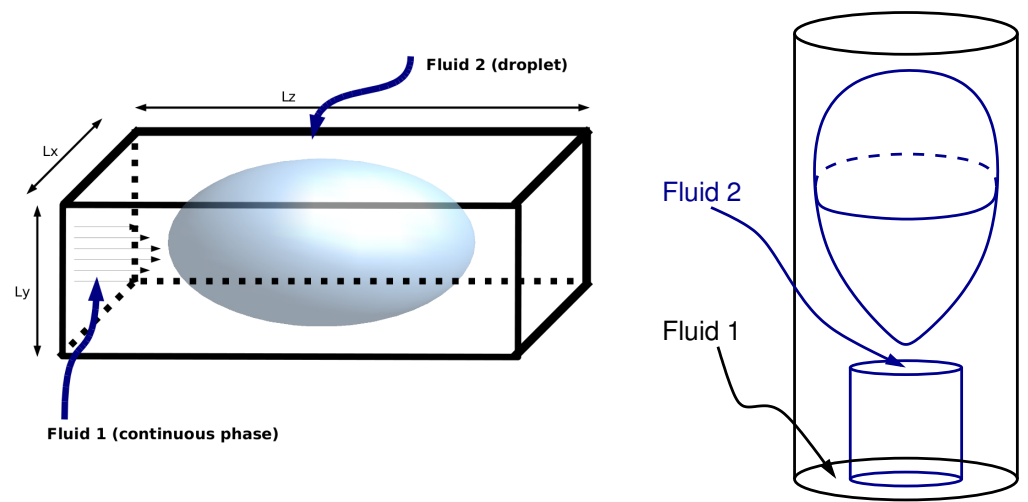

Fig. 1 Geometry of rectangular (on the left) and cylindrical (on the right) channels

3. Solve the Stokes equation (3)-(4) for (u, $p$ ) with $\kappa$ and $\mathbf{n}$ obtained by step 2 .

4. Update $\phi$ by solving (9).

5. If needed, apply a redistanciation procedure on $\phi$ in order to ensure $|\nabla \phi|=1$ (see [6]).

6. Iterate step 2-5 for each time step.

The Stokes system (3)-(4) is discretized on a staggered mesh using a finite volume scheme. The divergence free condition is ensured by an augmented Lagrangian algorithm. The transport equation (9) is discretized by a fifth order WENO scheme [5].

Note that in microfluidics, surface tension is preponderant and a specific stability condition derived in [2] is used :

$$
\Delta t=\min \left(c_{1} \frac{\Delta x}{\|\mathbf{u}\|_{\infty}}, c_{2} \frac{\eta}{\sigma} \Delta x\right)
$$

where $\Delta t$ is the time step and $\Delta x$ is the space step. The constant $c_{1}$ is linked to the classical CFL condition and depends only on the scheme used to discretize the advection equation. The constant $c_{2}$ is associated to the constraint induced by the surface tension term that is discretized explicitly. In our microfluidics applications, $c_{2}=4$ leads to stable computations (see [3]).

We use a penalization method [1] to take into account the spatial structure of the coaxial cylindrical channels shown on the right of Figure 1. Numerical approach for this axisymmetric framework is presented in [9]. 


\section{Numerical results}

\subsection{Results in 3D axisymmetric channels}

The first test case (Fig. 2) concerns the simulation of droplets creation by injecting a fluid into another, thanks to the "injector" geometry of figure 1 (right) where the radius of the external capillary is $R=300 \mu \mathrm{m}$. The internal capillary of length $R$, thickness $50 \mu \mathrm{m}$ and centered at $r=75 \mu \mathrm{m}$ is modelled by a penalization term. Two fluids have a surface tension $\sigma=33 \cdot 10^{-3} \mathrm{~N} / \mathrm{m}$. In the internal jet, the viscosity is $\eta_{2}=30.10^{-2}$ Pa.s and in the external capillary, the viscosity is $\eta_{1}=55.10^{-3}$ Pa.s. Parabolic profiles are used for the injection velocity at the inlet, with a maximum of $u_{2}=0.07 \mathrm{~m} / \mathrm{s}$ in the internal tube and $u_{1}=0.01 \mathrm{~m} / \mathrm{s}$ in external tube. The section $R$ of the computational domain is discretized with 30 cells. The jet breaks up because of Rayleigh instability.
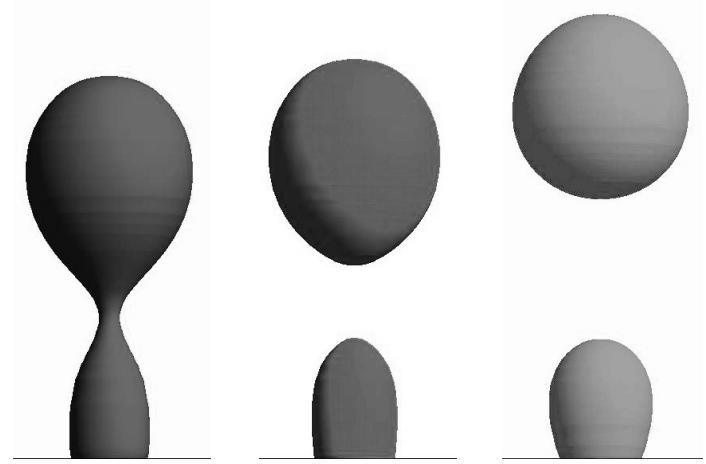

Fig. 2 Droplet creation with an axisymmetric jet. Time increases from left to right.

In the second case (Fig. 3), we then present two examples of flows that exist inside a microdroplet by showing the velocity field in the droplet frame of reference. We use an external capillary which is of radius $R=60 \mu \mathrm{m}$. The fluid inside the droplet has a viscosity $\eta_{2}=2.10^{-2}$ Pa.s and the viscosity of the continuous phase is $\eta_{1}=4.10^{-2}$ Pa.s. The injection speed considered is $0.2 \mathrm{~m} / \mathrm{s}$ and $0.1 \mathrm{~m} / \mathrm{s}$ respectively for the two numerical simulations. It can be observed that when the velocity is small, the droplet shape is more spherical and a central recirculation region develops towards the front of the droplet. This is due to the increasing influence of the surface tension compared to the driving flow. 

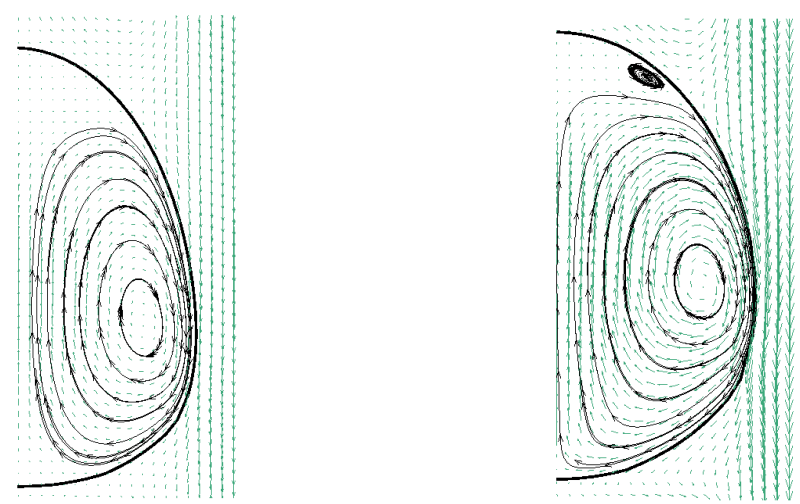

Fig. 3 Hydrodynamics in a cylindrical channel : the droplet is propelled with an injection speed of $0.2 \mathrm{~m} / \mathrm{s}$ on the left and $0.1 \mathrm{~m} / \mathrm{s}$ on the right.

\subsection{Results in $3 D$ rectangular channels}

We now present comparative simulations in the 3D cartesian geometry of the left of Figure 1 with $L_{x}=L_{y}=150 \mu \mathrm{m}$. Again, the viscosity is $\eta_{2}=2.10^{-2} \mathrm{~Pa}$.s in the droplet and $\eta_{1}=4.10^{-2}$ Pa.s elsewhere and the surface tension between them is $\sigma=33.10^{-3} \mathrm{~N} / \mathrm{m}$. Figures 4 and 5 show a droplet with an injection speed of 0.2 $\mathrm{m} / \mathrm{s}$ and $0.1 \mathrm{~m} / \mathrm{s}$, respectively. A bigger droplet is also shown on Figure 6. The influence of the injection speed is the same as in the axisymmetric case if the global evolution of the shape and the recirculation zones are taken into account (see Fig. 3 and 4,5). But differences definitely appear when it comes to compare droplets shapes and induced streamlines. First, when looking at a cross section (with respect to the direction of the flow), droplets in a square channel are not spherical - contrary to the cylindrical case - as it can be seen on the back of droplets of Figures 4 and 5 and even more clearly on the slices numbered 3 and 4 on Figure 7 which are at the back of the droplet of Figure 6. On the right of Figure 6, the velocity field in a $(\mathrm{x}, \mathrm{y})$ section shows a typical fully $3 \mathrm{D}$ behaviour, which is only seen in rectangular configuration : eight vortexes are present inside the droplet near its boundary that correspond to the fluid that focuses at the center. It is clearly not an axisymmetric phenomenon. At the boundary of the channel, the flow is deviated in the direction of the corners. The conservation of the flow rates implies that the liquid has to escape in the longitudinal direction through the four corners. This fully $3 \mathrm{D}$ effect is due to the rectangular confinement. 

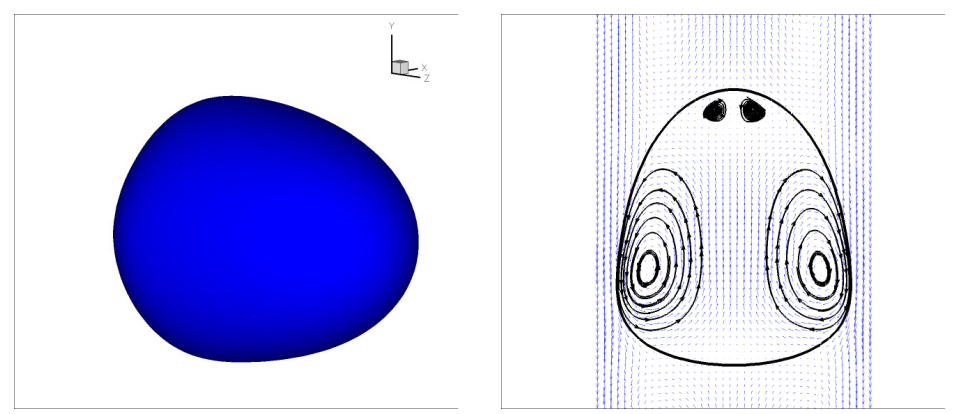

Fig. 4 Droplet hydrodynamics in a 3D cartesian configuration with an injection speed of $0.2 \mathrm{~m} / \mathrm{s}$.
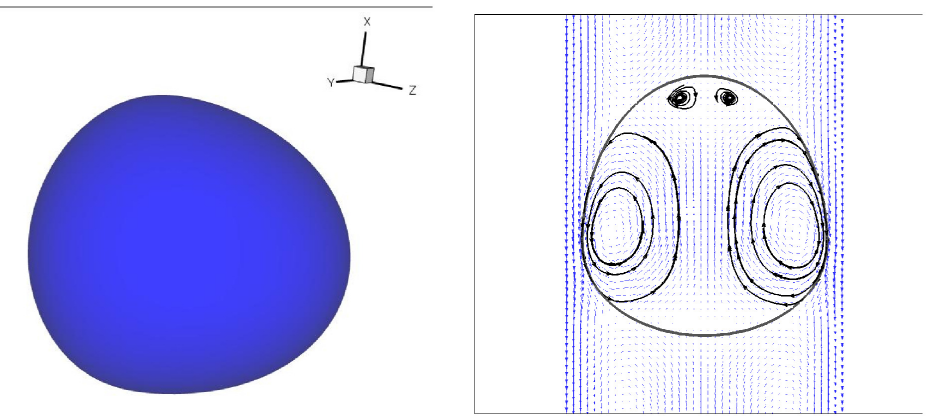

Fig. 5 Droplet hydrodynamics in a 3D cartesian configuration with an injection speed of $0.1 \mathrm{~m} / \mathrm{s}$.
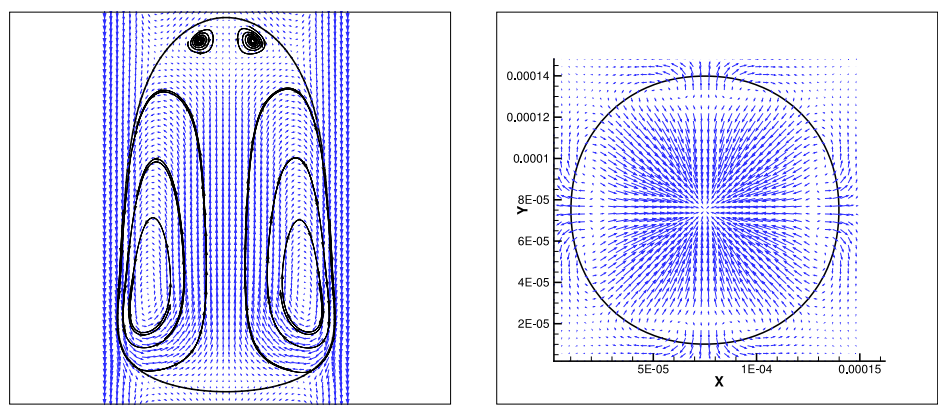

Fig. 6 A bigger droplet with an injection speed of $0.2 \mathrm{~m} / \mathrm{s}$ : velocity field respectively in the droplet's frame of reference (slice in plane (x,z) on the left) and in the global one (slice in (x,y) on the right).

\section{Conclusions}

In this note, we present various 3D dynamics in microdroplets thanks to a numerical method designed to handle flows driven by surface tension and pressure gradient 


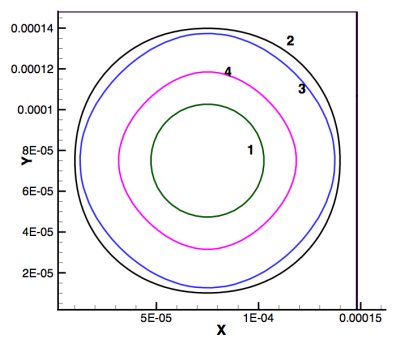

Fig. 7 Slices numbered from 1 to 4 (from the nose of the droplet to the back) in plane (x,y).

in a microfluidic framework. It appears that cylindrical and rectangular microchannels used in practical applications induced clearly different hydrodynamics mainly due to effect of rectangular confinement. This will be further studied by comparing jet stabilities in these two kinds of geometries, as well as mixing in droplets, and compared with equivalent physical experiments in microchannels [4].

\section{References}

1. Angot, P., Bruneau, C.H., Fabrie, P.: A penalization method to take into account obstacles in incompressible viscous flows. Numerische Mathematik 81(4), 497-520 (1999)

2. Galusinski, C., Vigneaux, P.: Level-Set method and stability condition for curvature-driven flows. C. R. Acad. Sci. Paris, Ser. I 344(11), 703-708 (2007)

3. Galusinski, C., Vigneaux, P.: On stability condition for bifluid flows with surface tension : application to microfluidics. Journal of Computational Physics 227(12), 6140-6164 (2008)

4. Guillot, P.: Ecoulements de fluides immiscibles dans un canal submillimétrique: Stabilité et application à la rhéologie. Ph.D. thesis, Université Bordeaux 1 (2006)

5. Jiang, G.S., Peng, D.: Weighted ENO schemes for Hamilton-Jacobi equations. SIAM Journal of Scientific Computing 21(No. 6), p. 2126-2143 (2000)

6. Osher, S., Fedkiw, R.: Level Set Methods and Dynamic Implicit Surfaces, Applied Mathematical Sciences, vol. 153. Springer (2003)

7. Sethian, J.A.: Level Set Methods and Fast Marching Methods - Evolving interfaces in computational geometry, fluid mechanics, computer vision and materials science, Cambridge Monographs on Applied and Computational Mathematics, vol. 3, second edn. Cambridge University Press (1999)

8. Sussman, M., Smereka, P., Osher, S.: A level set approach for computing solutions to incompressible two-phase flow. J. Comput. Phys 114, p. 146-159 (1994)

9. Vigneaux, P.: An axisymmetric Level-Set method for microdroplets hydrodynamics. under review (2007) 